\title{
On energy harvesting from ambient vibration
}

\author{
N.G. Stephen* \\ School of Engineering Sciences, Mechanical Engineering, University of Southampton, Highfield, Southampton SO17 1 BJ, UK
}

Received 9 May 2005; received in revised form 6 October 2005; accepted 10 October 2005

Available online 15 December 2005

\begin{abstract}
Future MEMS devices will harvest energy from their environment. One can envisage an autonomous condition monitoring vibration sensor being powered by that same vibration, and transmitting data over a wireless link; inaccessible or hostile environments are obvious areas of application. The base excitation of an elastically mounted magnetic seismic mass moving past a coil, considered previously by several authors, is analysed in detail. The amplitude of the seismic mass is limited in any practical device and this, together with the magnitude and frequency of the excitation define the maximum power that can be extracted from the environment. The overall damping coefficient (part of which is mechanical) is associated with the harvesting and dissipation of energy and also the transfer of energy from the vibrating base into the system. It is shown that net energy flow from the base through the damper is positive (negative) for $\omega>\omega_{n}\left(\omega<\omega_{n}\right)$, but is zero when $\omega=\omega_{n}$. The mechanical part of the damper cannot contribute more power than it dissipates and is neutral, at best, when $\omega / \omega_{n} \rightarrow \infty$. Maximum power is delivered to an electrical load when its resistance is equal to the sum of the coil internal resistance and the electrical analogue of the mechanical damping coefficient, which differs from what has been claimed. A highly damped system has the advantage of harvesting energy over a wider band of excitation frequencies on either side of the natural frequency, is smaller, but will harvest marginally less power. One possible strategy for variable amplitude excitation is proposed.
\end{abstract}

(C) 2005 Elsevier Ltd. All rights reserved.

\section{Introduction}

There has been much recent interest in the concept of low-power microelectromechanical systems (MEMS) that are able to scavenge, or harvest, energy from their operating environment. For example, one can envisage an autonomous condition monitoring sensor, measuring vibration level, being powered by the very same vibration, and transmitting data over a wireless link. Assuming the available power to be small, one envisages that the sensor would need to be intelligent — harvesting and storing energy for a period of time, before taking and transmitting a reading, followed by a further period of harvesting. Such devices could be employed in potentially hostile or inaccessible environments, and would require little or no maintenance.

A mechanical model of such a device, consisting of the base excitation of an elastically mounted seismic mass has received much attention, and was first proposed by Williams and Yates [1]. While the elastic mount is typically modelled as a coil spring, in practice a cantilever beam would provide both the flexibility and

*Tel.: + 442380592359 ; fax: + 442380593230 .

E-mail address: ngs@soton.ac.uk. 


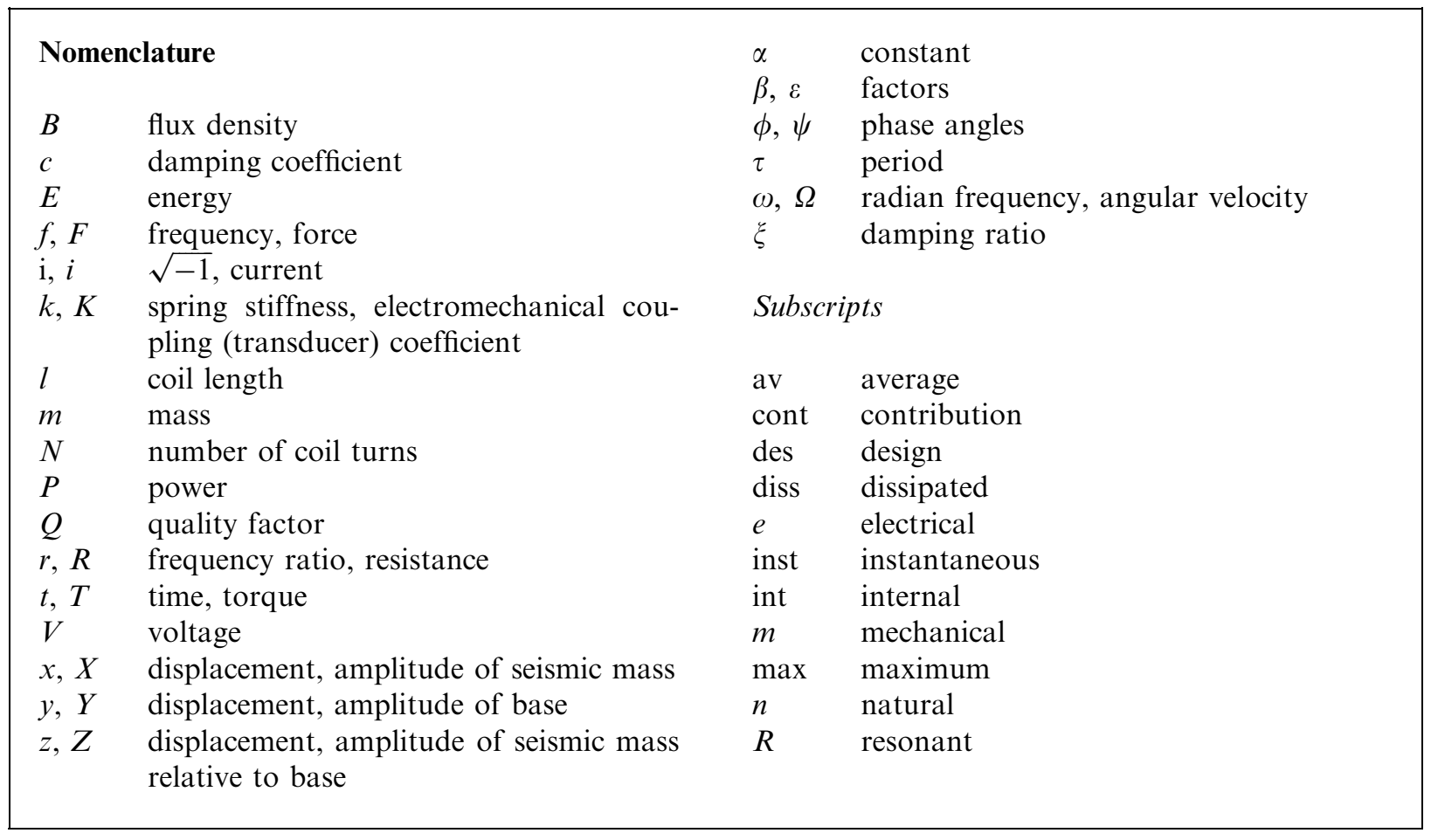

necessary constraint of the seismic mass without the need for bearing surfaces which are prone to high friction and hence heat generation at small scales. The means of energy extraction may consist of a magnetic seismic mass moving past a coil, or the beam may be fabricated from piezoelectric material. As far as the mechanical vibration is concerned, the combination of mechanical and electrical damping is treated as equivalent to linear viscous damping (proportional to velocity). This is a fair representation for the electromagnetic conversion considered by Williams and Yates [1], less so for a piezoelectric conversion; nevertheless, many interesting conclusions may be drawn from this simple model. Prototype devices have been described by El-Hami et al. [2], Roundy et al. [3], and Williams et al. [4]. Related applications and reviews may be found in Refs. [5-11].

Here, the theory governing the mechanical (and less so electrical) behaviour of such devices is examined in detail. Although the base excitation of a single degree of freedom (dof) spring-mass-damper model is perfectly well understood - it is described in most, if not all, typical undergraduate textbooks on vibration (see, for example Ref. [12]) - many of the derived expressions can be written in a bewildering variety of forms, and it is shown how easy it is to draw erroneous conclusions, as some authors have done. Typically, these relate to the role of damping. The overall damping coefficient (of which the mechanical damping is a part) plays an ambiguous role, being associated not only with the harvesting and dissipation of energy, but also the transfer of energy from the vibrating base into the system. It is shown that net energy flow from the base through the damper is positive (negative) for $\omega>\omega_{n}\left(\omega<\omega_{n}\right)$, but is zero when $\omega=\omega_{n}$. It is also shown that the mechanical component of the overall damping cannot contribute more energy than it dissipates; this is consistent with experimental evidence by Williams et al. [4] indicating that the operation of such a device in a vacuum is beneficial. Potential design features of future devices are proposed. One must recognise that in any practical device, the amplitude of the seismic mass is limited to some maximum value and this, together with the magnitude and frequency of the excitation (the operating environment), define the maximum power that can be extracted from the environment. A highly damped system has the advantage of harvesting energy over a wider band of excitation frequencies on either side of the natural frequency, is potentially smaller, but will harvest marginally less power. The theory is developed first for an alternating force applied directly to the seismic mass, before considering the more involved case of base excitation; it transpires that while the dimensionless average powers for the two cases are different in form, their dependence on frequency and damping ratios is identical. It is noted that three different resonant frequencies can be defined for the two cases 
considered, but maximum power is extracted from the environment when the frequency of excitation is equal to the undamped natural frequency, irrespective of the damping ratio.

Having considered the effect of total damping - consisting of useful energy harvested, together with electrical and mechanical dissipation - the effect of these is considered individually. The concept of impedance matching for maximum power delivery to an electrical load is addressed. Again, various authors have made conflicting claims, some suggesting that the impedance of the electrical load should be matched to the source impedance of the generator (coil), others that power delivery is optimised when mechanical and electrical damping ratios are equal. It is shown that maximum power is delivered to the electrical load when its resistance is equal to the sum of the coil internal resistance and the electrical analogue of the mechanical damping coefficient (here such matching is termed EDAM). Practically, this is equivalent to the matching of electrical and mechanical damping, if the coil resistance is small. However, mechanical damping should be as small as possible, which defines a minimum electrical damping coefficient dependent on the size of the device.

A possible strategy to harvest energy from a variable amplitude environment is also discussed, suggesting that controllable electrical damping is advantageous, so the ideal sensor would be intelligent with regard to both the harvesting of the energy, and its subsequent use.

Last, it is noted that the analysis is directed towards present generation devices that may be loosely described as millimetre-centimetre sized; however, as size reduction leads towards what may be described truly as MEMS [13], where characteristic lengths are typically less than $1 \mathrm{~mm}$ but greater than $1 \mu \mathrm{m}$ (and are fabricated using integrated-circuit batch-processing technologies), miniaturisation has the effect of increasing the ratio of surface area $\left(\right.$ length $\left.{ }^{2}\right)$ to mass $\left(\right.$ length $\left.^{3}\right)$ so surface effects, typically viscous forces, become more important. This suggests the reduction of mechanical damping by operating such a device in partial vacuum; in turn, one can envisage the breakdown of continuum viscous assumptions, and the necessity of stochastic descriptions (the Langevin equation) for the dynamics of the seismic mass. Such issues are not addressed here.

\section{Theory}

\subsection{Direct mass excitation}

The 1dof spring-mass-damper shown in Fig. 1 is subject to a sinusoidal force applied directly to the mass; the governing equation of motion is [12]

$$
m \ddot{x}+c \dot{x}+k x=F \sin \omega t ;
$$

dot denotes differentiation with respect to time. Multiply by velocity $\dot{x}$, and rearrange as

$$
\dot{x} F \sin \omega t=c(\dot{x})^{2}+m \dot{x} \ddot{x}+k x \dot{x}
$$

or

$$
\dot{x} F \sin \omega t=c(\dot{x})^{2}+\frac{\mathrm{d}}{\mathrm{d} t}\left(\frac{m \dot{x}^{2}}{2}+\frac{k x^{2}}{2}\right) .
$$

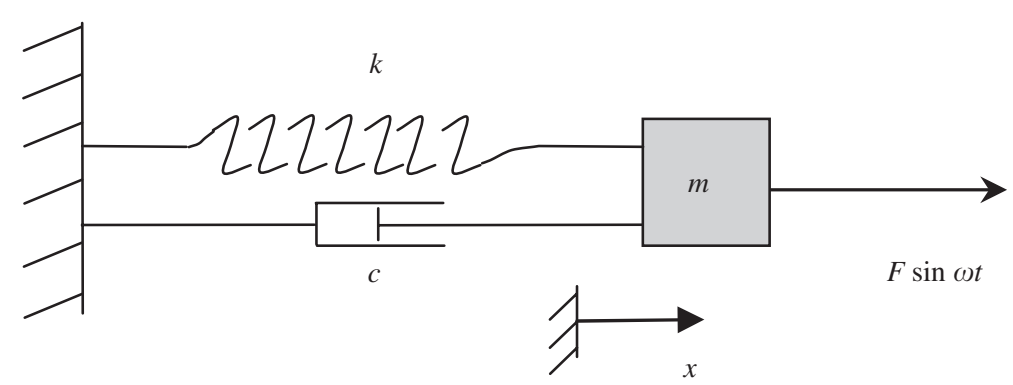

Fig. 1. Direct force excitation of spring-mass-damper. 
This represents conservation of power: in words, Eq. (2) states that the instantaneous power into the system is equal to the instantaneous power dissipated and/or absorbed by the damper plus the time rate of change (presumably increase) of the sum of the kinetic and strain energies. Of immediate note, is that in the absence of damping $(c=0)$ the power dissipated or absorbed is obviously zero, and the power input goes entirely to the build-up of energy (and amplitude) of the spring-mass oscillator; more importantly, the system will never achieve a steady-state condition.

The steady-state response is

$$
x=X \sin (\omega t-\phi),
$$

where

$$
X=\frac{F}{\sqrt{\left(k-\omega^{2} m\right)^{2}+c^{2} \omega^{2}}}
$$

and the phase angle $\phi=\tan ^{-1}\left(c \omega /\left(k-\omega^{2} m\right)\right)$. The resonant frequency, defined as the frequency for which the forced response has its maximum, is $\omega_{R}=\omega_{n} \sqrt{1-2 \xi^{2}}$, while the natural frequency is $\omega_{n}=\sqrt{k / m}$. The damping ratio is $\xi=c /\left(2 \sqrt{\mathrm{km}}=c / 2 m \omega_{n}\right)$.

The instantaneous power absorbed by the damper is $c(\dot{x})^{2}$ and, for $\dot{x}=\omega X \cos (\omega t-\phi)$, this becomes

$$
P_{\text {inst }}=c \omega^{2} X^{2} \cos ^{2}(\omega t-\phi) \text {. }
$$

The energy harvested per cycle is

$$
E_{\text {cycle }}=c X^{2} \omega^{2} \int_{0}^{\tau=2 \pi / \omega} \cos ^{2}(\omega t-\phi) \mathrm{d} t=\pi c \omega X^{2},
$$

where $\tau=2 \pi / \omega$ is the period of the cycle. Now define the average power flow as $P_{\mathrm{av}}=E_{\text {cycle }} / \tau$, to give

$$
P_{\mathrm{av}}=c \omega^{2} X^{2} / 2 \text {. }
$$

If one substitutes for the amplitude $X$ from Eq. (4), then the average power becomes

$$
P_{\mathrm{av}}=\frac{c \omega^{2} F^{2}}{2\left(\left(k-\omega^{2} m\right)^{2}+c^{2} \omega^{2}\right)} .
$$

The latter can be expressed in dimensionless form by dividing both the numerator and denominator by $k^{2}$, and re-arranging to give

$$
\bar{P}_{\mathrm{av}}=\frac{P_{\mathrm{av}}}{F^{2} /\left(\omega_{n} m\right)}=\frac{\xi\left(\omega / \omega_{n}\right)^{2}}{\left(1-\left(\omega / \omega_{n}\right)^{2}\right)^{2}+\left(2 \xi \omega / \omega_{n}\right)^{2}} .
$$

Now differentiate this expression with respect to the frequency ratio $r=\left(\omega / \omega_{n}\right)$, set $\partial \bar{P}_{\mathrm{av}} / \partial r=0$ to give $2 \xi r\left(1-r^{4}\right)=0$, and $r= \pm 1, \pm \mathrm{i}$, where $\mathrm{i}=\sqrt{-1}$. Thus, despite the amplitude of vibration having its maximum value at the resonant frequency $\omega_{R}=\omega_{n} \sqrt{1-2 \xi^{2}}$, maximum power flow occurs for $\omega=\omega_{n}$, when Eq. (9) reduces to

$$
P_{\mathrm{av}}=F^{2} /\left(4 \xi \omega_{n} m\right) .
$$

At this stage it is tempting, but quite wrong, to conclude that $P_{\mathrm{av}} \rightarrow \infty$ as $\xi \rightarrow 0$. Setting the damping ratio to zero implies that one is not harvesting any energy. Moreover, as is clear from Eq. (2), the system is no longer in a steady-state, so the assumptions leading to this false conclusion are no longer valid. The opposite, and equally invalid, conclusion may be reached as follows: first, one must recognise that in any practical device seeking to harvest energy in this way, the amplitude of the seismic mass will be limited to some maximum value, say $X_{\max }$. Eq. (7) then becomes, for $\omega=\omega_{n}$,

$$
P_{\mathrm{av}}=c \omega_{n}^{2} X_{\max }^{2} / 2
$$

from which one might be tempted to conclude that the damping coefficient should be as large as possible. The reality, however, is that as damping is increased, the amplitude $X$ would become less than $X_{\max }$, unless the 
amplitude of the force $F$ increases accordingly. Thus the conclusion $P_{\mathrm{av}} \rightarrow \infty$ as $c \rightarrow \infty$ would also require the quite impossible condition that $F \rightarrow \infty$. On the other hand, it is perfectly clear from Eq. (11) that the power extracted is zero, when damping is zero.

A third approach for a force of specified magnitude $F$ and frequency $\omega$, and for some maximum allowable displacement $X_{\max }$, is to (part) substitute from Eq. (4) into Eq. (7) to give

$$
P_{\mathrm{av}}=\frac{c \omega^{2} F X_{\max }}{2 \sqrt{\left(k-\omega^{2} m\right)^{2}+c^{2} \omega^{2}}}
$$

or in dimensionless form

$$
\bar{P}_{\mathrm{av}}=\frac{P_{\mathrm{av}}}{\omega F X_{\max }}=\frac{\xi\left(\omega / \omega_{n}\right)}{\sqrt{\left(1-\left(\omega / \omega_{n}\right)^{2}\right)^{2}+\left(2 \xi \omega / \omega_{n}\right)^{2}}} .
$$

The condition for a maximum is that $\partial \bar{P}_{\mathrm{av}} / \partial r=\partial \bar{P}_{\mathrm{av}} / \partial \xi=0$, and both of these are satisfied for $r=1$, that is when the frequency of excitation is equal to the undamped natural frequency, not the resonant frequency. Substituting $\omega=\omega_{n}$ into Eqs. (12) or (13) gives

$$
P_{\mathrm{av}}=\omega_{n} F X_{\max } / 2 .
$$

It should be emphasised that this expression represents the maximum flow of power into the device, i.e. extracted from the alternating force. The maximum useful electrical power that could be harvested (delivered to an electrical load) would be subject to further constraints; this is considered in Section 3 for the case of base excitation. At first sight, the fact that Eq. (14) is independent of the damping ratio suggests that this amount of power can be harvested even when damping ratio is zero. A similar anomaly has been noted previously by Langley [14], who showed that that possibility can be discounted because, as above, one would not have steady-state conditions; rather, when damping ratio is zero the expression $P_{\mathrm{av}}=\omega_{n} F X / 2$ still represents the power flow into the device, but this goes towards increasing the amplitude of the oscillator. Eq. (14) can be found more directly by integrating the left-hand side of Eq. (2) to give the power flow into the device as $\pi F X \sin \phi$ per cycle, and average power as $\omega F X \sin \phi / 2$; this achieves its maximum value for $\omega=\omega_{n}$ when the phase angle $\phi$ is equal to $90^{\circ}$, irrespective of the damping ratio.

A surface plot of the non-dimensional power as expressed by Eq. (13) is shown in Fig. 3, and the following observations are made:

(a) Suppose that the magnitude of the force $F$ is small. The displacement amplitude $X$ of the seismic mass is related to this force by the expression $X=F /\left(2 m \xi \omega_{n}^{2}\right)$, which is obtained by setting $\omega=\omega_{n}$ in Eq. (4), so in turn $X$ will be small, perhaps less than the maximum value. By reducing the damping ratio, $X$ can be allowed to increase to its maximum value, so the device will absorb the maximum possible power from the environment; the latter will be small, however, because force $F$ is small.

(b) On the other hand, suppose that the magnitude of the force $F$ is large. In turn, $X$ would be large, possibly exceeding the allowable value. Damping would now have to be increased, so that the displacement of the seismic mass does not exceed its maximum value; the power absorbed from the environment would still be expressed by Eq. (14), but would now be large because the force $F$ is large. Thus one role of damping is to maintain $X$ equal to $X_{\max }$.

(c) It is clear from the above, that the power that can be absorbed is limited by $X_{\max }$, so this should be as large as possible in any potential device, notwithstanding that if the device is intended to be small, then $X_{\max }$ in turn will be small. Power is also proportional to the magnitude of the force, so this should be as large as possible. On the other hand, $P_{\text {av }} \rightarrow 0$ as $F \rightarrow 0$. This is the situation that holds when damping ratio goes to zero - for a force of fixed magnitude, the displacement amplitude tends to infinity at $\omega=\omega_{n}$ : this is the familiar concept of resonance. An alternative interpretation is that the force magnitude required to maintain displacement amplitude equal to its maximum value, goes to zero at $\omega=\omega_{n}$. So again, there is no anomaly regarding Eq. (14) being independent of damping ratio $\xi$.

(d) If the force is small, then light damping results in a highly tuned system, capable of harvesting its maximum value only at, or very close to, a frequency ratio of unity. If the force is large, not only will the maximum power be proportionately larger, so too will the damping ratio, and the device would be capable of 


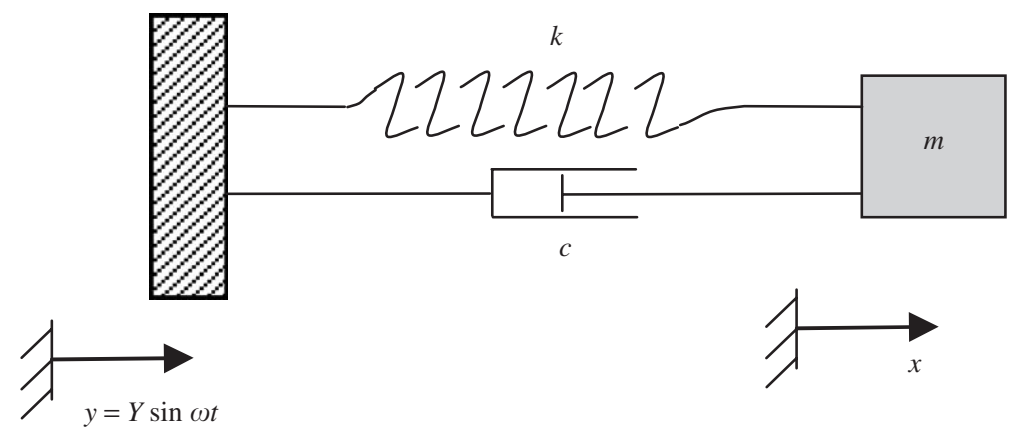

Fig. 2. Base excitation of spring-mass-damper.

extracting power close to the theoretical maximum value over a range of excitation frequencies either side of the natural frequency.

(e) Last, suppose that one had a force of variable magnitude; the ideal device would, in turn, have variable damping, indeed exactly the amount necessary to maintain the seismic mass displacement amplitude at its maximum value. This suggests the need for the device to be intelligent - to extract precisely the amount of energy necessary to maintain displacement amplitude at its maximum allowable value.

\subsection{Base excitation}

Referring to Fig. 2, the governing differential equation is

$$
m \ddot{x}+c(\dot{x}-\dot{y})+k(x-y)=0 .
$$

Multiplication by $\dot{x}$, and re-arrangement leads to the expression

$$
k(y-x) \dot{y}+c(\dot{y}-\dot{x}) \dot{y}=c(\dot{x}-\dot{y})^{2}+m \ddot{x} \dot{x}+k(x-y)(\dot{x}-\dot{y})
$$

or

$$
k(y-x) \dot{y}+c(\dot{y}-\dot{x}) \dot{y}=c(\dot{x}-\dot{y})^{2}+\frac{\mathrm{d}}{\mathrm{d} t}\left(\frac{m \dot{x}^{2}}{2}+\frac{k(x-y)^{2}}{2}\right) .
$$

Again, this is an expression of conservation of power: the instantaneous power into the system is equal to the power absorbed by the damper plus the time rate of increase of the sum of the kinetic and strain energies. As with direct mass excitation, in the absence of damping the power goes entirely towards the build-up of energy (and amplitude) of the spring-mass oscillator, with no steady-state being achieved. The damping coefficient will consist of mechanical and electrical contributions, and may be expressed as $c=c_{m}+c_{e}$. In contrast with Eq. (2), the damping coefficient appears on both sides of Eq. (16), so the mechanical contribution $c_{m}$ seemingly plays a beneficial role in the transfer of energy from the environment into the device, while simultaneously dissipating energy. However, as will be seen in Section 2.2.1, the energy per cycle contributed by the damper can be both positive and negative, and is zero when the frequency of excitation is equal to the natural frequency. Moreover, the mechanical component cannot contribute more energy than it dissipates.

Eq. (15) can be solved for the absolute displacement of the seismic mass, or for its displacement relative to the moving base. For the latter case, set $z=x-y$, and for harmonic base excitation $y=Y \sin \omega t$, one has

$$
m \ddot{z}+c \dot{z}+k z=m \omega^{2} Y \sin \omega t .
$$

This equation is identical in form to Eq. (1) if one replaces $F$ by $m \omega^{2} Y$, and $x$ by $z$. Accordingly, the steadystate solution is

$$
z=Z \sin (\omega t-\phi)
$$




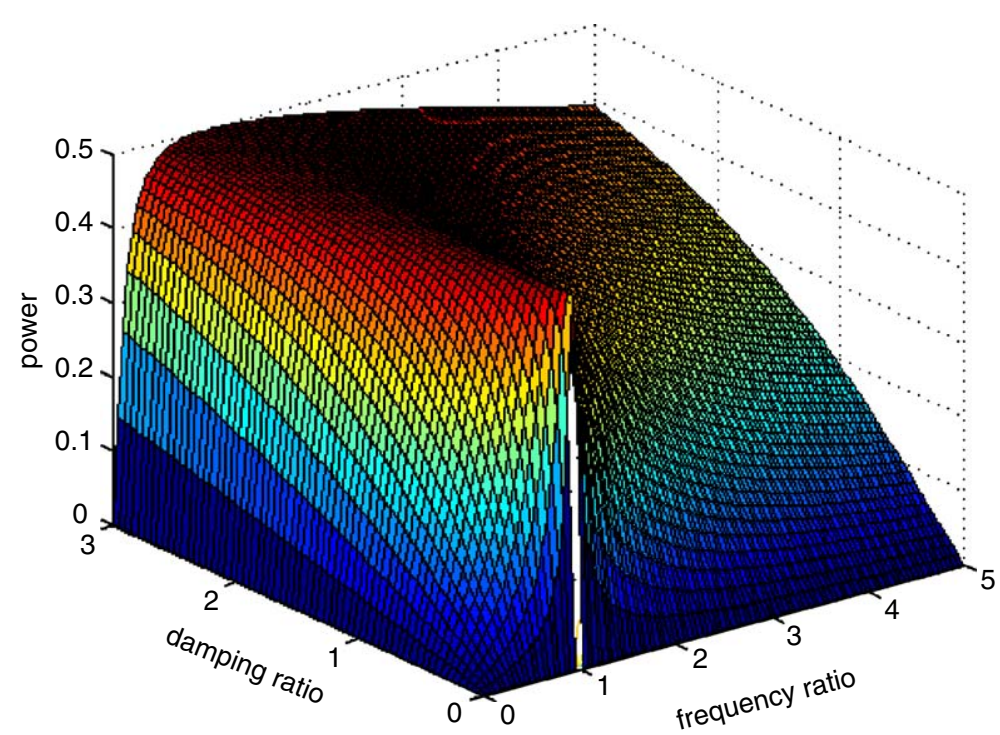

Fig. 3. Variation of dimensionless power with frequency ratio and damping ratio for both direct mass, and base excitation.

where

$$
Z=\frac{m \omega^{2} Y}{\sqrt{\left(k-\omega^{2} m\right)^{2}+c^{2} \omega^{2}}}
$$

and the phase angle is the same as for direct mass excitation. The absolute displacement of the seismic mass can then be calculated as $x=y+z$, although in practice it is easier to approach Eq. (15) afresh, using complex algebra [12]. The steady-state solution is

$$
x=X \sin (\omega t-\psi)
$$

where

$$
X=\frac{\sqrt{k^{2}+c^{2} \omega^{2}}}{\sqrt{\left(k-\omega^{2} m\right)^{2}+c^{2} \omega^{2}}}
$$

with phase angle

$$
\psi=\tan ^{-1}\left(\frac{m c \omega^{3}}{k\left(k-\omega^{2} m\right)+c^{2} \omega^{2}}\right) .
$$

One may define two different resonant frequencies for base excitation, both of which differ from that of direct mass excitation; the seismic mass has its maximum (absolute) displacement amplitude $X$ when excited at frequency [12]

$$
\omega_{R}=\frac{\omega_{n}}{2 \xi}\left(\sqrt{1+8 \xi^{2}}-1\right)^{1 / 2}
$$

This frequency is always less than the undamped natural frequency; employing the binomial expansion, the radical in the above expression can be written as

$$
\sqrt{1+8 \xi^{2}}=1+4 \xi^{2}-8 \xi^{4}+32 \xi^{8}-\cdots
$$

Assuming damping ratio to be small, then ignoring terms higher than $\xi^{2}$ leads to $\omega_{R}=\omega_{n}$; ignoring terms higher than $\xi^{4}$ leads to $\omega_{R}=\omega_{n} \sqrt{1-2 \xi^{2}}$, while ignoring terms higher than $\xi^{8}$ leads to 


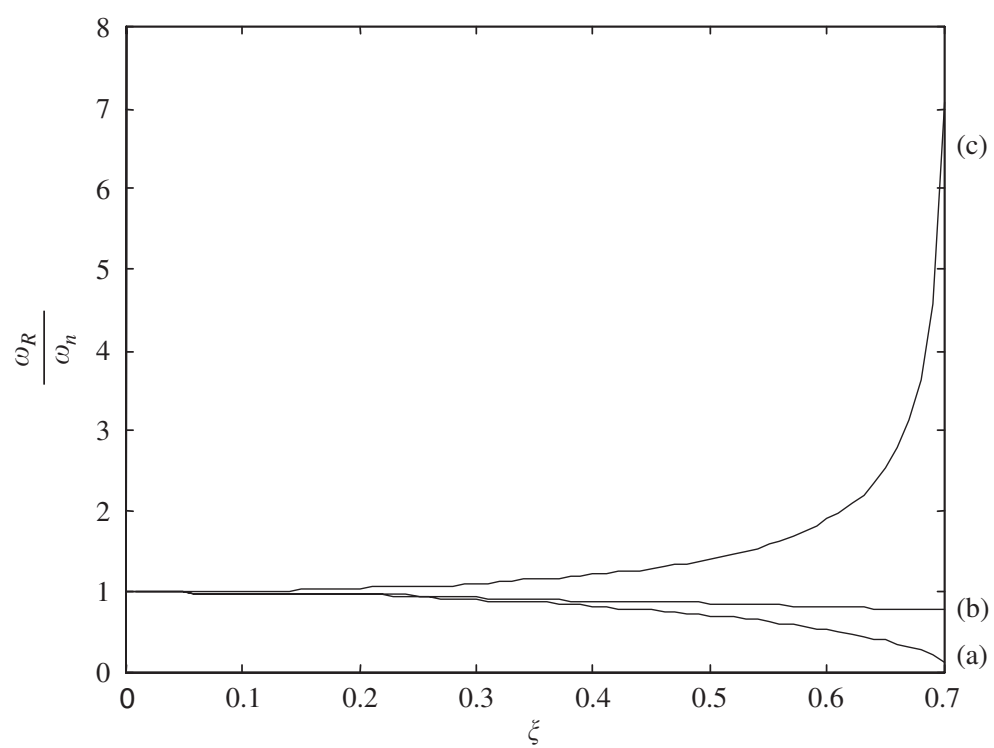

Fig. 4. Variation of ratio of resonant to natural frequency, $\omega_{R} / \omega_{n}$, with damping ratio $\xi$. The curves in ascending magnitude are (a) direct mass excitation $\omega_{R} / \omega_{n}=\sqrt{1-2 \xi^{2}}$; (b) base excitation, maximum absolute response of the seismic mass $\omega_{R} / \omega_{n}=\left(\sqrt{1+8 \xi^{2}}-1\right)^{1 / 2} /(2 \xi)$; (c) base excitation, maximum response relative to the base $\omega_{R} / \omega_{n}=1 / \sqrt{1-2 \xi^{2}}$.

$\omega_{R}=\omega_{n} \sqrt{1-2 \xi^{2}+8 \xi^{6}}$. Thus one concludes that the difference between this resonant frequency, and that for direct mass excitation, is small at least when damping ratio is small. Indeed, as seen in Fig. 4, the difference is practically indistinguishable for $\xi<0.3$. On the other hand, the seismic mass has its maximum displacement amplitude relative to the base, $Z$, when excited at frequency [12]

$$
\omega_{R}=\frac{\omega_{n}}{\sqrt{1-2 \xi^{2}}}
$$

which is always greater than the undamped natural frequency. This characteristic is also shown in Fig. 4, where the damping ratio range extends only to $\xi=1 / \sqrt{2}$; for larger values, there is no resonant maximum amplitude for two of the three defined resonant frequencies.

The average power, in terms of the relative displacement amplitude of the seismic mass, is identical to Eq. (7) derived in Section 2.1, so long as one replaces $X$ by $Z$, and is

$$
P_{\mathrm{av}}=c \omega^{2} Z^{2} / 2=\xi m \omega_{n} \omega^{2} Z^{2} .
$$

Substituting for $Z$ from Eq. (19), we also have

$$
P_{\mathrm{av}}=\frac{c m^{2} \omega^{6} Y^{2}}{2\left(\left(k-\omega^{2} m\right)^{2}+c^{2} \omega^{2}\right)}
$$

or in near dimensionless form

$$
P_{\mathrm{av}}=\frac{m \xi Y^{2}\left(\omega / \omega_{n}\right)^{3} \omega^{3}}{\left(1-\left(\omega / \omega_{n}\right)^{2}\right)^{2}+\left(2 \xi \omega / \omega_{n}\right)^{2}}=\frac{m \xi Y^{2}\left(\omega / \omega_{n}\right)^{6} \omega_{n}^{3}}{\left(1-\left(\omega / \omega_{n}\right)^{2}\right)^{2}+\left(2 \xi \omega / \omega_{n}\right)^{2}}
$$

which is the expression derived by Williams and Yates [1], El-Hami et al. [2] and Mitcheson et al. [11], amongst others; note that the expression given by Roundy et al. [3] contains a typographical error. Again, it is very easy to draw false conclusions from these expressions. In the anticipation that the power would be largest when the system is close to resonance, set $\omega=\omega_{n}$ in Eq. (28), to give

$$
P_{\mathrm{av}}=\omega_{n}^{3} m Y^{2} /(4 \xi) \text {. }
$$


It is tempting, but quite wrong, to conclude that $P_{\mathrm{av}} \rightarrow \infty$ as $\xi \rightarrow 0$, for exactly the reasons described in Section 2.1-one would not have steady-state conditions. However, this was the conclusion reached by Williams and Yates [1], claiming that "In principle, a damping factor of zero would generate infinite power at resonance, but in practice this is not possible." More recently Mitcheson et al. [11] also claimed that the optimal damping ratio was zero for $\omega=\omega_{n}$, suggesting that "the power extracted can be increased without limit by decreasing $\xi$ ", but qualified this by noting that zero damping would lead to excessive relative displacement of the seismic mass. On the other hand, from Eq. (26), one might conclude that $P_{\text {av }} \rightarrow \infty$ as $\xi \rightarrow \infty$, but this would also require that $Y \rightarrow \infty$, which again is impossible.

Further, one can see from the above the reasoning behind the claim that the electrical and mechanical damping ratios should be equal. If one writes $\xi=\xi_{m}+\xi_{e}$, and then regard the electrical part as potentially useful, then Eq. (28) with $\omega$ set equal to $\omega_{n}$ becomes

$$
P_{\mathrm{av} \mathrm{elec}}=\frac{m \xi_{e} Y^{2} \omega_{n}^{3}}{4\left(\xi_{m}+\xi_{e}\right)^{2}}
$$

now set $\mathrm{d} P_{\text {av elec }} / \mathrm{d} \xi_{e}=0$ to give $\xi_{e}=\xi_{m}$; these steps are valid, as the electrical and mechanical damping ratios are independent. This was the conclusion reached in Refs. [3,4]: for maximum power flow into the electrical domain, one requires matching between mechanical and electrical loads. However, it should be noted that what is really required is not maximum power into the electrical domain (where there are further inevitable losses), rather maximum power flow into the electrical load. One may partition the electrical damping ratio as $\xi_{e}=\xi_{e \text { load }}+\xi_{e \text { loss }}$, when the useful average power to the electrical load may be written as

$$
P_{\text {av elec load }}=\frac{m \xi_{e \text { load }} Y^{2} \omega_{n}^{3}}{4\left(\xi_{m}+\xi_{e \text { loss }}+\xi_{e \text { load }}\right)^{2}} ;
$$

add the mechanical damping loss to the electrical loss to give the total loss as $\xi_{\text {loss }}=\xi_{m}+\xi_{e \text { loss }}$, when Eq. (31) becomes

$$
P_{\text {av elec load }}=\frac{m \xi_{e \text { load }} Y^{2} \omega_{n}^{3}}{4\left(\xi_{\text {loss }}+\xi_{e \text { load }}\right)^{2}} .
$$

It is tempting to set $\mathrm{d} P_{\text {av } e \text { load }} / \mathrm{d} \xi_{e \text { load }}=0$, to give $\xi_{e \text { load }}=\xi_{\text {loss }}$; however, this approach is invalid, as $\xi_{e \text { load }}$ and $\xi_{e \text { loss }}$ (and hence $\xi_{\text {loss }}$ ) are not independent, as will be seen in Section 3. Load matching must be applied within the domain to which power is being delivered, Ref. [15], and in the above, one is attempting to maximise electrical power from the viewpoint of the mechanical domain.

As with the case of direct mass excitation, a practical device will have a maximum displacement amplitude of the seismic mass relative to its housing, $Z_{\max }$, and the average power can be expressed as

$$
P_{\mathrm{av}}=\frac{c m \omega^{4} Y Z_{\max }}{2 \sqrt{\left(k-\omega^{2} m\right)^{2}+c^{2} \omega^{2}}}
$$

or in dimensionless form

$$
\bar{P}_{\mathrm{av}}=\frac{P_{\mathrm{av}}}{m \omega^{3} Y Z_{\max }}=\frac{\xi\left(\omega / \omega_{n}\right)}{\sqrt{\left(1-\left(\omega / \omega_{n}\right)^{2}\right)^{2}+\left(2 \xi \omega / \omega_{n}\right)^{2}}} .
$$

The right-hand side of the above is identical to that of Eq. (13), which means that the observations made for direct mass excitation are broadly transferable to the case of base excitation; again it must be emphasised that these observations pertain to the flow of energy from the environment into the device, not power delivery to an electrical load. In particular, it should be designed to operate at a frequency ratio $r=1$, that is $\omega=\omega_{n}$, when the average power flow into the device is $P_{\text {flow av }}=m \omega_{n}^{3} Y Z_{\max } / 2$ which again is independent of the damping ratio. Clearly, excitation amplitude $Y$ should be as large as possible, but this is a characteristic of the environment. The allowable excursion of the seismic mass $m$ within the device, and the mass itself, should also be large, notwithstanding that a small device inevitably implies that $Z_{\max }$ will be small. However, damping needs to be controllable according to the magnitude of $Y$. If the latter is small, then damping should be small 
to allow $Z$ to approach its maximum value; the power extracted will be small, because $Y$ is small. If $Y$ is large, then greater damping is required so that $Z$ does not exceed $Z_{\max }$ although now, more power is absorbed, because $Y$ is large. Again, the above expression does not suggest that power can be extracted for zero damping - suppose that the magnitude of $Y$ is variable; then, as damping approaches zero, so the required value of $Y$ necessary to maintain $Z$ equal to its maximum value also approaches zero and so, in turn, does $P_{\text {flow av }}$. From Fig. 3, it is again clear that the bandwidth of the device is greater when the damping ratio is large.

\subsubsection{Power flow contributions}

For base excitation $y=Y \sin \omega t$ and steady-state response $z=Z \sin (\omega t-\phi)$, with $z=x-y$, Eq. (16) becomes

$$
-k z \dot{y}-c \dot{z} \dot{y}=c \dot{z}^{2}
$$

Integrating over a cycle and noting the results

$$
\int_{0}^{\tau=2 \pi / \omega} \cos ^{2}(\omega t-\phi) \mathrm{d} t=\pi / \omega, \quad \int_{0}^{\tau=2 \pi / \omega} \cos \omega t \cos (\omega t-\phi) \mathrm{d} t=\cos \phi \times \pi / \omega
$$

and

$$
\int_{0}^{\tau=2 \pi / \omega} \sin (\omega t-\phi) \cos \omega t \mathrm{~d} t=-\sin \phi \times \pi / \omega,
$$

leads to the energy relationship

$$
k \pi Y Z \sin \phi-c \omega \pi Y Z \cos \phi=c \omega \pi Z^{2},
$$

from which the following conclusions may be drawn:

(a) The phase angle $\phi$ falls within the range $0 \leqslant \phi \leqslant \pi$, so $\sin \phi \geqslant 0$ and the net energy contribution by the spring - the term involving $k$ on the left-hand side of Eq. (36) - cannot be negative.

(b) For $\omega<\omega_{n}$, the phase angle $\phi<\pi / 2$ so $\cos \phi$ is positive, and the net energy contribution by the damper - the term involving $c$ on the left-hand side of Eq. (36) - is negative.

(c) For $\omega=\omega_{n}$, the phase angle $\phi=\pi / 2$ so the net energy contribution by the damper over a cycle is zero. Eq. (36) reduces to $k Y=c \omega_{n} Z$, or $Z=k Y /\left(c \omega_{n}\right)=m \omega_{n} Y / c$, which is also what one finds from Eq. (19).

(d) For $\omega>\omega_{n}$, the phase angle $\phi>\pi / 2$ so $\cos \phi$ is negative, and the net energy contribution by the damper is positive. The spring and the damper make equal contributions when $k \sin \phi=-c \omega \cos \phi$, or $\tan \phi=-c \omega / k$; the general expression for the phase angle is $\tan \phi=c \omega /\left(k-\omega^{2} m\right)$, and equality requires that $\omega=\sqrt{2} \omega_{n}$. This explains, physically, the existence of a cross-over point at $\omega=\sqrt{2} \omega_{n}$, and the beneficial (adverse) effect of damping on vibration isolation for $\omega<\sqrt{2} \omega_{n}\left(\omega>\sqrt{2} \omega_{n}\right)$. When $\omega \gg \omega_{n}$, the phase angle $\phi \rightarrow \pi$ irrespective of the damping ratio, so $\sin \phi \rightarrow 0$ and the energy contributed by the spring approaches zero. Eq. (36) then reduces to $Y=Z$. If the damping coefficient is partitioned as $c=c_{e}+c_{m}$, then the energy per cycle contributed by the mechanical damping is $E_{m}$ cont $=-c_{m} \omega \pi Y Z \cos \phi$, while that dissipated is $E_{m \text { diss }}=c_{m} \omega \pi Z^{2}$. It is straightforward to show that $E_{m \text { cont }} / E_{m \text { diss }}=1-\left(\omega / \omega_{n}\right)^{-1}$, in which case the mechanical component of the damping will always contribute less energy that it dissipates; it is neutral, at best, in the limit $\left(\omega / \omega_{n}\right) \rightarrow \infty$.

\section{Electrical power generation}

The arguments presented so far have made no real distinction between desirable damping - electrical energy harvesting from the device - and the inevitable mechanical and electrical losses. Also, they have focused on power flow from the environment into the device, rather than the delivery of useful power to an electrical load. These issues are now discussed in more detail for the case of base excitation. In common with most previous investigations, inductance is ignored. The simplest form of electromechanical coupling, as typified by a permanent-magnet DC motor, is assumed. In this model, torque $T$ is related to current $i$ according to $T=K i$, where the transducer coefficient $K$ has units $\mathrm{Nm} / \mathrm{amp}$, and voltage (back emf) $V$ is related to angular velocity 

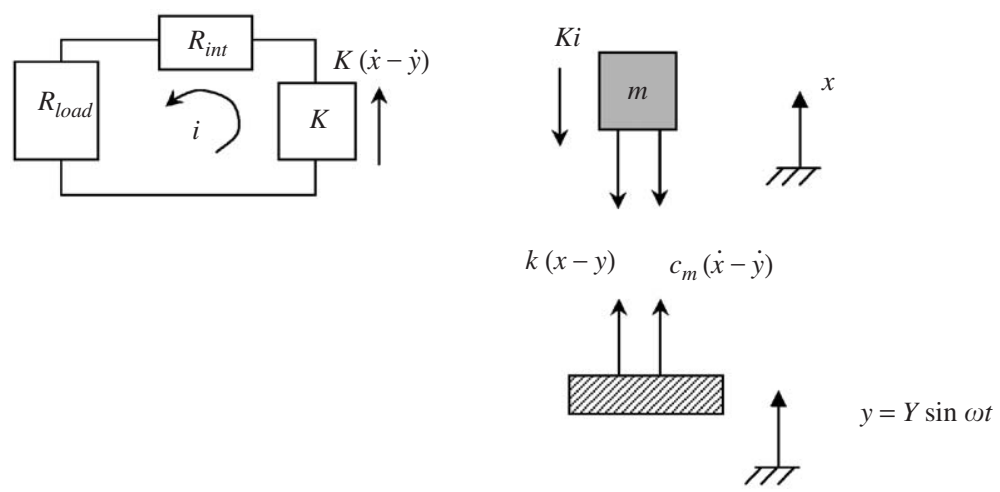

Fig. 5. Base excitation: free-body diagrams and electrical circuit.

$\Omega$ by the same coefficient as $V=K \Omega$, where the coefficient now has units volts $/ \mathrm{rad} / \mathrm{s}$; such transduction is $100 \%$ efficient. For the movement of the magnetic seismic mass past the coil, the above is modified such that force $F$ is proportional to current and voltage is proportional to relative translational velocity.

Referring to Fig. 5, Kirchhoff's voltage law for the electrical circuit gives

$$
i\left(R_{\text {load }}+R_{\text {int }}\right)-K(\dot{x}-\dot{y})=0
$$

while Newton's second law for the seismic mass gives

$$
-k(x-y)-c_{m}(\dot{x}-\dot{y})-K i=m \ddot{x} ;
$$

eliminating the current $i$, gives

$$
m \ddot{x}+c(\dot{x}-\dot{y})+k(x-y)=0,
$$

where the total damping coefficient is

$$
c=c_{m}+K^{2} /\left(R_{\mathrm{load}}+R_{\mathrm{int}}\right)
$$

this also defines the electrical damping coefficient as $c_{e}=K^{2} /\left(R_{\text {load }}+R_{\text {int }}\right)$. Note that the electromechanical coupling coefficient $K$ may be written as $K=N B l$ where $N$ is the number of coil turns, $B$ is the average magnetic flux density in the air-gap and $l$ is the coil length across the magnetic flux [5,6], so $K$ will be approximately linearly related to the internal resistance of the coil and one may write $K=\alpha R_{\text {int }}$, where $\alpha$ is a constant dependent on the construction of the coil.

For $\omega=\omega_{n}$, which is assumed in what follows, the seismic mass has displacement

$$
z=\frac{m \omega_{n} Y}{c} \sin \left(\omega_{n} t-\phi\right)=Z \sin \left(\omega_{n} t-\phi\right)
$$

so the amplitudes of the base excitation and the seismic mass are related as

$$
Z=m \omega_{n} Y / c=m \omega_{n} Y /\left(c_{e}+c_{m}\right) .
$$

The earlier statement that $Z_{\max }$ should be as large as possible is now qualified. From Eq. (42), the relationship between base and seismic mass amplitudes can be expressed as

$$
Z=\frac{m \omega_{n} Y}{c_{m}+K^{2} /\left(R_{\text {load }}+R_{\text {int }}\right)}=\frac{m \omega_{n} Y}{c_{m}+\alpha^{2} R_{\text {int }}^{2} /\left(R_{\text {load }}+R_{\text {int }}\right)} .
$$

For a given $Y$, one can always increase $Z$ by reducing $K$ until eventually the latter is zero. Assuming some lower limit on $c_{m}, Z$ would then have its maximum possible value, and maximum power will flow from the environment into the device; however no power is transferred into the electrical domain, rather it is dissipated entirely through mechanical damping. 
Delivery of power to an electrical load, and impedance matching, is now considered; from Eqs. (37) and (41), the current is

$$
i=\frac{K}{R_{\text {load }}+R_{\text {int }}} \omega_{n} Z \cos \left(\omega_{n} t-\phi\right)
$$

and as the overall damping coefficient increases, so $Z$ becomes smaller, and so too does the current. For a maximum excursion of the seismic mass $Z_{\max }$ (defined by the physical dimensions of the device), the current can be expressed as

$$
i=\frac{K}{R_{\text {load }}+R_{\text {int }}} \omega_{n} Z_{\max } \cos \left(\omega_{n} t-\phi\right) ;
$$

the instantaneous power transferred to the electrical load is then

$$
P_{\text {load inst }}=i^{2} R_{\text {load }}=\frac{K^{2} R_{\text {load }} \omega_{n}^{2} Z_{\text {max }}^{2}}{\left(R_{\text {load }}+R_{\text {int }}\right)^{2}} \cos ^{2}\left(\omega_{n} t-\phi\right),
$$

and the average value is

$$
P_{\text {load av }}=\frac{K^{2} R_{\text {load }} \omega_{n}^{2} Z_{\text {max }}^{2}}{2\left(R_{\text {load }}+R_{\text {int }}\right)^{2}} .
$$

The power can also be expressed in terms of the product of $Y$ and $Z_{\max }$, as

$$
P_{\text {load av }}=\frac{K^{2} R_{\text {load }} m \omega_{n}^{3} Y Z_{\text {max }}}{2\left(R_{\text {load }}+R_{\text {int }}\right)\left(c_{m}\left(R_{\text {load }}+R_{\text {int }}\right)+K^{2}\right)},
$$

or in terms of the amplitude of the base excitation $Y$, as

$$
P_{\text {load av }}=\frac{K^{2} R_{\text {load }} m^{2} \omega_{n}^{4} Y^{2}}{2\left(c_{m}\left(R_{\text {load }}+R_{\text {int }}\right)+K^{2}\right)^{2}}
$$

Further, if one rearranges Eq. (49) as

$$
P_{\text {load av }}=\frac{\left(K^{2} R_{\text {load }} /\left(R_{\text {load }}+R_{\text {int }}\right)^{2}\right) m^{2} \omega_{n}^{4} Y^{2}}{2\left(c_{m}+K^{2} /\left(R_{\text {load }}+R_{\text {int }}\right)\right)^{2}},
$$

and identify $c_{e \text { load }}=K^{2} R_{\text {load }} /\left(R_{\text {load }}+R_{\text {int }}\right)^{2}$ and $c_{e \text { loss }}=K^{2} R_{\text {int }} /\left(R_{\text {load }}+R_{\text {int }}\right)^{2}$, so that $c_{e}=c_{e \text { load }}+c_{e \text { loss }}=$ $K^{2} /\left(R_{\text {load }}+R_{\text {int }}\right)$, then Eq. (50) reduces to

$$
P_{\text {load av }}=\frac{c_{e \text { load }} m^{2} \omega_{n}^{4} Y^{2}}{2\left(c_{m}+c_{e \text { loss }}+c_{e \text { load }}\right)^{2}} .
$$

Writing $c=2 m \omega_{n} \xi$ for each of the contributors to the total damping (electrical load and loss, and mechanical), this becomes equal to Eq. (32); in particular it becomes clear that the two electrical damping ratios are not independent - a change in any parameter of the electrical system $\left(K, R_{\text {load }}\right.$ or $\left.R_{\text {int }}\right)$ will affect both of the electrical damping ratios.

Various load matching conditions are now considered. The requirement for maximum power transfer, $\mathrm{d} P_{\text {load av }} / \mathrm{d} R_{\text {load }}=0$, yields different conditions when applied to Eqs. (47-49).

\subsection{Electrical resistance matching}

Application to Eq. (47), assuming $Z_{\max }$ and $R_{\text {int }}$ to be constant, leads to the familiar requirement of electrical resistance matching, which is $R_{\text {load }}=R_{\text {int }}$. This derivation is perfectly analogous to that for a nonreactive purely electrical circuit consisting of internal and load resistances, and a voltage source of constant magnitude $V=K \omega_{n} Z_{\max } / \sqrt{2}$. However, this approach is incorrect, as it implies that for base excitation of fixed amplitude $Y$, the seismic mass excursion is equal to $Z_{\max }$ irrespective of the amount of damping present, which is untrue (rather it is given by Eq. (42)). It would be valid only if changes in the magnitude of $R_{\text {load }}$ conspired with other changes - for example, the magnitude of the mechanical damping coefficient, $c_{m}$ - in such 
a way as to keep the overall damping ratio constant. With such matching, the average power delivered to the electrical load may be expressed as

$$
P_{\text {load av }}^{(1)}=\frac{K^{2} R_{\text {int }} m^{2} \omega_{n}^{4} Y^{2}}{2\left(2 c_{m} R_{\text {int }}+K^{2}\right)^{2}}
$$

Applying $\mathrm{d}\left(P_{\text {load av }}\right) / \mathrm{d} R_{\text {load }}=0$ to Eq. (48) leads to the expression $R_{\text {load }}^{2}=R_{\text {int }}^{2}+R_{\text {int }} K^{2} / c_{m}$, but this approach is again incorrect because it assumes that $Z$ is equal to $Z_{\max }$, irrespective of the overall damping.

\subsection{Domain matching}

Setting the electrical damping coefficient equal to the mechanical, implies $c_{m}=c_{e}=K^{2} /\left(R_{\mathrm{load}}+R_{\text {int }}\right)$, or $R_{\text {load }}=K^{2} / c_{m}-R_{\text {int }}$. With such matching, the average power delivered to the load may be expressed as

$$
P_{\text {load av }}^{(2)}=\frac{\left(K^{2} / c_{m}-R_{\text {int }}\right) m^{2} \omega_{n}^{4} Y^{2}}{8 K^{2}} .
$$

\subsection{Electrical domain analogue matching (EDAM)}

The correct approach is to apply $\mathrm{d}\left(P_{\text {load av }}\right) / \mathrm{d} R_{\text {load }}=0$ to Eq. (49), when one finds the relationship

$$
R_{\text {load }}=R_{\text {int }}+K^{2} / c_{m}
$$

This is what one would expect from the concept of resistance matching within the electrical domain, when it includes the electrical analogue of the mechanical damping, in order to transfer maximum power to an electrical load, Ref. [15]. With such matching, the average power delivered to the electrical load may be expressed as

$$
P_{\text {load av }}^{(3)}=\frac{K^{2} m^{2} \omega_{n}^{4} Y^{2}}{8 c_{m}\left(c_{m} R_{\text {int }}+K^{2}\right)} .
$$

One can see that this is greater than the power delivered with simple electrical resistance matching by noting that $P_{\text {load av }}^{(3)} / P_{\text {load av }}^{(1)}=1+K^{4} /\left(4 c_{m} R_{\text {int }}\left(c_{m} R_{\text {int }}+K^{2}\right)\right)$, which is clearly greater than unity. Similarly, for comparison with domain matching, one can derive $P_{\text {load av }}^{(3)} / P_{\text {load av }}^{(2)}=1+c_{m}^{2} R_{\text {int }}^{2} /\left(c_{m} R_{\text {load }}\left(c_{m} R_{\text {int }}+K^{2}\right)\right)$ where $R_{\text {load }}=K^{2} / c_{m}-R_{\text {int }}$; again this is clearly greater than unity.

This maximum average power transferred to the electrical load, Eq. (55) can be expressed as

$$
P_{\text {load av max }}=\frac{m^{2} \omega_{n}^{4} Y^{2}}{8 c_{m}}\left(1-\frac{R_{\text {int }}}{R_{\text {load }}}\right)=\frac{m \omega_{n}^{3} Y^{2}}{16 \xi_{m}}\left(1-\frac{R_{\text {int }}}{R_{\text {load }}}\right)
$$

and a variety of other forms, such as

$$
P_{\text {load av max }}=\frac{m \omega_{n}^{3} Y Z_{\text {max }}}{4}\left(\frac{R_{\text {load }}-R_{\text {int }}}{R_{\text {load }}+R_{\text {int }}}\right)=\frac{m \omega_{n}^{3} Y Z_{\max }}{4}\left(\frac{c_{e}}{c_{m}}\right)=\frac{P_{\text {flow av }}}{2}\left(\frac{c_{e}}{c_{m}}\right) .
$$

The power dissipated within the coil internal resistance is

$$
P_{\text {int }}=\frac{m \omega_{n}^{3} Y Z_{\max }}{4} \frac{R_{\text {int }}}{R_{\text {load }}}\left(\frac{R_{\text {load }}-R_{\mathrm{int}}}{R_{\text {load }}+R_{\text {int }}}\right)=\frac{m \omega_{n}^{3} Y Z_{\mathrm{max}}}{4} \frac{R_{\text {int }}}{R_{\text {load }}}\left(\frac{c_{e}}{c_{m}}\right),
$$

while the power dissipated within the mechanical domain is

$$
P_{\mathrm{mech}}=\frac{m \omega_{n}^{3} Y Z_{\mathrm{max}}}{4}\left(\frac{R_{\mathrm{load}}+R_{\mathrm{int}}}{R_{\mathrm{load}}}\right)
$$


It is straightforward to show that power is conserved, that is

$$
P_{\text {load av max }}+P_{\text {int }}+P_{\text {mech }}=P_{\text {flow av }} .
$$

The power delivered to the electrical load can be related also to the flow of power into the device from the environment as

$$
P_{\text {load av max }}=\frac{P_{\text {flow av }}}{2}\left(\frac{R_{\text {load }}-R_{\text {int }}}{R_{\text {load }}+R_{\text {int }}}\right)=\frac{P_{\text {flow av }}}{2}\left(\frac{c_{e}}{c_{m}}\right)=\frac{P_{\text {flow av }}}{2}\left(\frac{\xi_{e}}{\xi_{m}}\right),
$$

from which it is clear that the internal resistance $R_{\text {int }}$ should be as small as possible. From the relationship $K=\alpha R_{\text {int }}$, one concludes that the ideal electromechanical transducer should have large $\alpha$ (high flux density) with internal resistance as small as possible. The maximum power transferred to the electrical load will always be less than one-half of that flowing into the device. Given the relationship implicit within Eq. (61), i.e. $c_{e} / c_{m}=\left(R_{\text {load }}-R_{\text {int }}\right) /\left(R_{\text {load }}+R_{\text {int }}\right)$, the impedance matching condition EDAM leads to the conclusion that the electrical damping ratio will be less than the mechanical. In the ideal limit $R_{\text {int }} \rightarrow 0$ (with $\alpha \rightarrow \infty$, so that $K$ is finite) the matching condition reduces to $R_{\text {load }}=K^{2} / c_{m}$, which implies resistance matching between the electrical and the mechanical domains, or $c_{e}=c_{m}$, which is the condition claimed by Roundy et al. [3] and Williams et al. [4]. However, it has also been shown that mechanical damping always has an adverse effect, so a second ideal limit is $c_{m} \rightarrow 0$. The EDAM condition, Eq. (54), then suggests that the load resistance should increase without limit, implying that $c_{e} \rightarrow 0$; in turn, the relative seismic mass displacement $Z \rightarrow \infty$. In reality, the minimum damping coefficient is constrained by the size of the device, from Eq. (42), as $c_{e}+c_{m} \geqslant m \omega_{n} Y / Z_{\max }$, and in the ideal limit that mechanical damping is zero, $c_{e} \geqslant m \omega_{n} Y / Z_{\max }$.

\section{Relationship with other published work}

Despite claiming that damping ratio should be small in order to harvest the maximum amount of power, Williams and Yates [1] presented theoretical predictions of generated power for a range of frequencies of excitation, amplitude of vibration source, and seismic mass displacement (relative to the base) for a damping ratio $\xi=0.3$. In fact these represent power extracted from the environment, rather than power delivered to an electrical load, for the reasons stated above; the numerical values presented in Table 1 of Ref. [1] are in agreement with Eq. (34), as they must - the relationship between the amplitude of the base excitation $Y$, and the excursion of the seismic mass, $Z$, from Eq. (42), is fixed as $Z / Y=1 /(2 \xi)=Q$ for $\omega=\omega_{n}$.

On the other hand, El-Hami et al. [2] report measured and calculated usable power (themselves in very good agreement) significantly less than the maximum values predicted in the present paper. For example, at a frequency of $f=322 \mathrm{~Hz}$, base amplitude of $Y=25 \times 10^{-6} \mathrm{~m}$, seismic mass $m=0.51 \times 10^{-3} \mathrm{~kg}$ and seismic mass displacement (it is not clear whether this is absolute or relative displacement) of $0.94 \times 10^{-3} \mathrm{~m}$, a useful electrical power of $0.53 \mathrm{~mW}$ was reported. First, note that the ratio of the seismic mass displacement to base amplitude is $\left(0.94 \times 10^{-3}\right) /\left(25 \times 10^{-6}\right)=37.6$, which describes a very lightly damped system; moreover, this indicates that any ambiguity as to whether the reported seismic mass displacement is relative or absolute, is unimportant regarding the following observations, as the difference between the two is at most $\pm 1 / 37.6= \pm 2.66 \%$. From Eq. (34), the power flow from the environment into the device would be $49.63 \mathrm{~mW}$, which is approximately one-hundred times greater than the electrical power delivered to the load $(0.53 \mathrm{~mW})$. A $Q$ factor of 37.6 indicates an overall damping ratio of $\xi=1 /(2 \times 37.6)=0.0133$. The magnitude of the mechanical damping ratio was unknown, but estimated in Ref. [2] as $\xi_{m}=0.013$, in which case the electrical damping ratio would be $\xi_{e}=0.0003$. This implies that the majority of the power flowing into the device, as expressed by Eq. (34), is being dissipated as a mechanical loss rather than being harvested as useful electrical power; amplitude $Z$ was being maximised, rather than delivered power. If the system was designed to achieve mechanical and electrical resistance matching, the overall damping ratio would be $\xi=0.026$, the displacement amplitude of the seismic mass would be (more or less) halved, as would the flow of power from the environment into the device, but the power delivered to the electrical domain would be approximately $12.7 \mathrm{~mW}$, a 24 fold increase. 


\section{Behaviour of an intelligent device}

Now consider the possible behaviour of an intelligent device in response to variable amplitude base excitation: we suppose that the device has been designed for maximum power transfer into the electrical domain $\left(\xi_{e}=\xi_{m}\right.$, or $\left.R_{\text {load }}+R_{\text {int }}=K^{2} / c_{m}\right)$ for some target excitation amplitude $Y_{0}$, with a maximum seismic mass excursion, from Eq. (42), of

$$
Z_{\max }=Y_{0} /\left(4 \xi_{e}\right)
$$

this defines the minimum electrical damping ratio. The (design) power flow into the electrical domain may be written as

$$
P_{\mathrm{des}}=\frac{c_{e}}{2\left(c_{e}+c_{m}\right)} m \omega_{n}^{3} Y_{0} Z_{\max }=m \omega_{n}^{3} Y_{0} Z_{\max } / 4,
$$

which is one-half of the power flowing from the base into the device. Now suppose that $Y_{0}$ reduces to $9 Y_{0} / 10$; the magnitude of the coil internal resistance and the mechanical damping are fixed so unless the electrical load resistance increases (that is, the electrical damping decreases), $Z$ will also reduce to $9 Z_{\max } / 10$, and the power transferred to the electrical domain reduces to

$$
P_{e}=(9 / 10)^{2} m \omega_{n}^{3} Y_{0} Z_{\max } / 4=0.81 \times P_{\text {des }},
$$

which is a $19 \%$ reduction. Instead, allow the electrical damping coefficient to reduce from $c_{e}=c_{m}$ to $c_{e}=\beta c_{m}$ where $\beta$ is less than unity, and determine the required value of $\beta$ to allow $Z$ to resume its maximum value; this requires, from Eq. (43)

$$
Z_{\max }=\frac{m \omega_{n} Y_{0}}{2 c_{m}}=\frac{m \omega_{n}\left(9 Y_{0} / 10\right)}{c_{m}+\beta c_{m}},
$$

which leads to $\beta=0.8$. The power transferred to the electrical domain is now, from Eq. (63) $P_{e}=\left(0.8 c_{m} /\left(2\left(0.8 c_{m}+c_{m}\right)\right)\right) m \omega_{n}^{3}(9 / 10) Y_{0} Z_{\max }=0.8 \times P_{\text {des }}$. From this example, one concludes that seeking to maintain $Z$ at its maximum is actually counter-productive. Instead, suppose we allow $Z$ to reduce to $\varepsilon Z_{\max }$ by reducing the electrical damping coefficient from $c_{e}=c_{m}$ to $c_{e}=\beta c_{m}$ where both $\varepsilon$ and $\beta$ are less than unity, and seek to improve upon the power, according to Eq. (64). First, one has

$$
\varepsilon Z_{\max }=\frac{\varepsilon m \omega_{n} Y_{0}}{2 c_{m}}=\frac{m \omega_{n}\left(9 Y_{0} / 10\right)}{c_{m}+\beta c_{m}}
$$

from which $\varepsilon=1.8 /(1+\beta)$. We now require

$$
P_{e}=\frac{\beta c_{m}}{2\left(\beta c_{m}+c_{m}\right)} m \omega_{n}^{3}(9 / 10) Y_{0}\left(\varepsilon Z_{\max }\right)>(9 / 10)^{2} m \omega_{n}^{3} Y_{0} Z_{\max } / 4
$$

which leads to the requirement $0>(1-\beta)^{2}$; this is impossible as the right-hand side of the inequality is clearly positive. One concludes that an intelligent device would be unable to ameliorate a reduction in base excitation amplitude.

On the other hand, suppose that $Y_{0}$ increases to $10 Y_{0} / 9$. The electrical damping coefficient must be increased to $c_{e}=\beta c_{m}$, with $\beta>1$, to maintain $Z$ equal to $Z_{\max }$; this requires

$$
Z_{\max }=\frac{m \omega_{n} Y_{0}}{2 c_{m}}=\frac{m \omega_{n}\left(10 Y_{0} / 9\right)}{c_{m}+\beta c_{m}},
$$

leading to $\beta=11 / 9$. The power delivered to the electrical domain is then

$$
P_{e}=\frac{(11 / 9) c_{m}}{2\left((11 / 9) c_{m}+c_{m}\right)} m \omega_{n}^{3}(10 / 9) Y_{0} Z_{\max }=(11 / 9) m \omega_{n}^{3} Y_{0} Z_{\max } / 4=(11 / 9) P_{\mathrm{des}},
$$

which is $22.2 \%$ greater than $P_{\text {des. }}$. Had the device been designed from the outset for a base amplitude of $10 Y_{0} /$ 9, with a commensurate increase in $Z_{\max }$ to $10 Z_{\max } / 9$, then the electrical power would have increased by the factor $(10 / 9)^{2}=1.235$, which is an increase of $23.5 \%$; however this design would be larger by the factor $10 / 9$. This sample calculation suggests that the power density of the device increases with the damping ratio, and is 
consistent with the statement by Roundy et al. [3], that "a highly damped system will only slightly underperform a lightly damped system provided that most of the damping is electrically induced." One concludes that an intelligent device would be able to respond to an increase in base excitation amplitude, and increase power delivered to the electrical domain accordingly. This suggests that for an environment in which the base excitation amplitude is of variable magnitude, the device should be designed for an amplitude toward the lower end of the range, but have the intelligence to increase electrical damping (by reducing $R_{\text {load }}$ ), and harvest more energy, for the higher amplitudes.

\section{Conclusions}

The extraction of energy from a vibrating environment has been analysed in some detail. For both direct mass (force) and base excitation, the maximum power flow into the device depends on the vigour of the environment (frequency and amplitude of force, or base) and the size of the device. The dependence on frequency and damping ratios is the same for both cases, with maximum flow when the frequency of excitation is equal to the undamped natural frequency. A highly damped system would extract energy over a wide bandwidth of frequencies either side of the natural frequency, would be smaller, but would extract marginally less power. For base excitation, the overall damping coefficient (part of which is mechanical) is associated with both the harvesting and dissipation of energy and also the transfer of energy from the vibrating base into the system. It is shown that net energy flow from the base through the damper is positive (negative) for $\omega>\omega_{n}\left(\omega<\omega_{n}\right)$, but is zero when $\omega=\omega_{n}$. The mechanical component of the overall damping dissipates more power than it contributes and is, at best, neutral when $\omega / \omega_{n} \rightarrow \infty$.

Employing the simplest form of electromagnetic transduction, as exemplified by a permanent magnetic DC motor/generator, the delivery of useful power to an electrical load is considered for the case of base excitation. Maximum power is delivered to an electrical load when its resistance is equal to the sum of the coil internal resistance and the electrical analogue of the mechanical damping coefficient, which differs from what has been claimed. At the same time, coil internal resistance and mechanical damping should be minimised, in which case a minimum overall damping coefficient is defined by the maximum excursion of the seismic mass. Greater electrical damping leads to a smaller device, and increased power density.

Possible behaviour of an adaptive device designed to operate within a variable amplitude environment has also been considered. It would be unable to ameliorate a reduction in base excitation amplitude, but could increase power delivered to the electrical domain in response to an increase in base excitation amplitude. This suggests that for an environment in which the base excitation amplitude is of variable magnitude, the device should be designed for an amplitude toward the lower end of the range, but have the intelligence to increase electrical damping (by reducing $R_{\text {load}}$ ), and harvest more energy, for the higher amplitudes.

\section{References}

[1] C.B. Williams, R.B. Yates, Analysis of a micro-electric generator for microsystems, Sensors and Actuators A 52 (1996) 8-11.

[2] M. El-Hami, P. Glynne-Jones, N.M. White, M. Hill, S. Beeby, E. James, A.D. Brown, J.N. Ross, Design and fabrication of a new vibration-based electromechanical generator, Sensors and Actuators A 92 (2001) 335-342.

[3] S. Roundy, P.K. Wright, J. Rabaey, A study of low level vibrations as a power source for wireless sensor nodes, Computer Communications 26 (2003) 1131-1144.

[4] C.B. Williams, C. Shearwood, M.A. Harradine, P.H. Mellor, T.S. Birch, R.B. Yates, Development of an electromagnetic microgenerator, IEE Proceedings: Circuits, Devices and Systems 148 (2001) 337-342.

[5] P. Glynne-Jones, M.J. Tudor, S.P. Beeby, N.M. White, An electromagnetic, vibration-powered generator for intelligent sensor systems, Sensors and Actuators A 110 (2004) 344-349.

[6] M. Mizuno, D.G. Chetwynd, Investigation of a resonance microgenerator, Journal of Micromechanics and Microengineering 13 (2003) 209-216.

[7] E.P. James, M.J. Tudor, S.P. Beeby, N.R. Harris, P. Glynne-Jones, J.N. Ross, N.M. White, An investigation of self-powered systems for condition monitoring applications, Sensors and Actuators A 110 (2004) 171-176.

[8] H.A. Sodano, D.J. Inman, G. Park, A review of power harvesting from vibration using piezoelectric materials, The Shock and Vibration Digest 36 (2004) 197-205.

[9] G.K. Ottman, H.F. Hofmann, A.C. Bhatt, G.A. Lesieutre, Adaptive piezoelectric energy harvesting circuit of wireless remote power supply, IEEE Transactions on Power Electronics 17 (2002) 669-676. 
[10] H.A. Sodano, G. Park, D.J. Inman, Estimation of electric charge output for piezoelectric energy harvesting, Strain 40 (2004) $49-58$.

[11] P.D. Mitcheson, T.C. Green, E.M. Yeatman, A.S. Holmes, Architectures for vibration-driven micropower generators, Journal of Microelectromechanical Systems 13 (2004) 429-440.

[12] S.S. Rao, Mechanical Vibrations, third ed., Addison-Wesley, Reading, MA, 1995.

[13] M. Gad-el-Hak (Ed.), The MEMS Handbook, CRC Press, Boca Raton, FL, 2002.

[14] R.S. Langley, Can an undamped oscillator dissipate energy?, Journal of Sound and Vibration 206 (1997) $624-626$.

[15] N.G. Stephen, On the maximum power transfer theorem within electromechanical systems, Sensors and Actuators A, under review. 